\title{
The death of aspirin for primary prevention-should aspirin be changed to a prescription only medication?
}

\author{
Aranyak Rawal ${ }^{1}$, Brandon Cave ${ }^{2}$, Devarshi Ardeshna ${ }^{3}$, David Hana ${ }^{4}$, Uzoma N. Ibebuogu ${ }^{2}$, \\ Rami N. Khouzam ${ }^{2}$ \\ ${ }^{1}$ Department of Internal Medicine-Pediatrics, University of Tennessee Health Science Center, Memphis, TN, USA; ${ }^{2}$ Division of Cardiovascular \\ Diseases, Department of Internal Medicine, University of Tennessee Health Science Center, Memphis, TN, USA; ${ }^{3}$ School of Medicine, University \\ of Tennessee Health Science Center, Memphis, TN, USA; ${ }^{4}$ Department of Internal Medicine, Kasr Al Ainy School of Medicine, Cairo, Egypt \\ Correspondence to: Aranyak Rawal, MD. Department of Internal Medicine-Pediatrics, University of Tennessee Health Science Center, 956 Court Ave., \\ Suite H316, Memphis, TN 38163, USA. Email: arawal1@uthsc.edu.
}

Submitted Jun 16, 2019. Accepted for publication Jun 28, 2019.

doi: $10.21037 / \mathrm{atm} .2019 .07 .05$

View this article at: http://dx.doi.org/10.21037/atm.2019.07.05

Since its discovery in the 1890 s, aspirin has become the most used drug worldwide (1). For decades, aspirin has been heralded for its use in the primary prevention of cardiovascular disease (CVD). More recently, the use of aspirin for primary prevention has become controversial, and subsequently, guidelines both in the United States and internationally have reflected this change. The United States Preventative Services Task Force (USPSTF) changed its recommendations in 2016 to recommend the use of Aspirin in adults with a $\geq 10 \% 10$-year CVD risk. Additionally, the level of recommendation was downgraded to a Grade B and Grade C for adults aged 50-59 and adults aged 60-69 years, respectively (2). This is a stark change from previous classification of Grade A in all men between the ages of 45-79 and all women between the ages of 55-79, regardless of pre-existing risk for developing CVD (3). Finally, the 2019 Guideline for the Primary Prevention of Cardiovascular Disease from the American Heart Association (AHA) and American College of Cardiology (ACC) recommends aspirin for adults aged 40-70 with an increased risk for CVD without a significant bleeding risk (4). Also, aspirin therapy appears to be losing ground for other indications as the ACC/AHA no longer includes aspirin in their recommendations for stroke prevention in patients with atrial fibrillation and $\mathrm{CHA}_{2} \mathrm{DS}_{2}-\mathrm{VASc}$ scores of 1 in men or 2 in women (5). Perhaps this signals a movement to coincide with our European colleagues whose guidelines do not recommend aspirin for primary prevention of CVD at all (6).
The driving factors for the changes, at least in the more recent case of ACC/AHA guidelines, appear to be driven by three excellent trials published in the past year. The ARRIVE, ASPREE and ASCEND trials demonstrated that aspirin has at best a modest cardio-protective effect in individuals without pre-existing CVD yet increase the bleeding risk (7-9). These trials all evaluated the efficacy of aspirin for primary prevention in three distinct populations. ARRIVE evaluated patients with an average or "medium" risk of developing CVD. ASPREE evaluated the benefit of aspirin in older patients for preventing CVD, and ASCEND evaluated aspirin's use in diabetics. Each study is briefly described below, but the overlying conclusion is that the benefit of aspirin is potentially outweighed by the risk of bleeding in patients. As the landscape for aspirin and its use for primary prevention changes, providers must navigate guidelines and trials to help guide patients on a case to case basis as to whether or not aspirin is correct for them. Moving to a prescription only model would facilitate discussion between providers and patients without established CVD who may buy the drug over the counter (OTC) about the risks and benefits of aspirin in their specific situation.

ARRIVE was a randomized, double blind, placebocontrolled trial that evaluated the efficacy of aspirin $v s$. placebo in men $>55$ years of age and women $>60$ years of age who were deemed to be at an average cardiovascular risk. It included a total of 12,546 patients who had an average 10 -year atherosclerotic cardiovascular disease 
(ASCVD) risk of $17.3 \%$ in the aspirin group and $17.4 \%$ in the placebo group. After a follow up of 60 months, the efficacy endpoint of time to first ischemic event (confirmed myocardial infarction, stroke, cardiovascular death, unstable angina, or transient ischemic attack) was reported in both intention-to-treat (ITT) and per-protocol (PP) analyses. Interestingly, there was a significant decrease in the rates of any myocardial infarction $(0.98 \%$ in the aspirin group vs. $1.84 \%$ in the placebo; $\mathrm{P}=0.0014)$ in the $\mathrm{PP}$ analysis. However, in the ITT analysis, there was no significant decrease in myocardial infarction between groups $1.52 \%$ in the aspirin group $v s .1 .78 \%$ in the placebo group; $\mathrm{P}=0.2325$ ). In the safety analysis, the aspirin group expectedly had a significantly higher rate of gastrointestinal (GI) bleeding ( $0.97 \%$ vs. $0.46 \% ; \mathrm{P}=0.007)$ compared to placebo. It can be argued that an ITT analysis is more indicative of real-world results as medication compliance can vary and more relevant to a practice-altering setting. The ITT analysis showed no significant decrease in any evaluated endpoint when aspirin was compared to placebo, while a safety analysis of the entire patient population showed a significant increase in risk of GI bleeding.

The ASPREE trial evaluated the utility of aspirin for primary prevention in patients that were aged 65 years or older and echoed the results of the ARRIVE trial. ASPREE was a randomized, double blind, placebo-controlled multicenter trial that compared aspirin with placebo in 19,114 patients for a median follow-up of 4.7 years. Similar to the ARRIVE trial, ASPREE had no significant decrease in cardiovascular events in patients randomized to aspirin but did have a significant increase in bleeding risk. ASPREE had similar cardiovascular event rates in the aspirin (10.7 per 1,000 person years) and placebo (11.3 per 1,000 person years) groups. In contrast to ARRIVE, which only reported rates of GI bleeding, ASPREE showed a significant increase in major hemorrhage in this older population. Major hemorrhage significantly increased in the aspirin group in comparison to placebo [8.6 events per 1,000 patient years for aspirin vs. 6.2 events per 1,000 patient years for placebo; hazard ratio (HR) 1.38 ; $95 \%$ confidence interval (CI) 1.18 1.62; $\mathrm{P}<0.001]$. Further classification of major hemorrhage revealed the majority were GI in nature and a significant increase in intracranial bleeding. ASPREE further bolstered the evidence of the major bleeding risk associated with aspirin, without evidence to support its cardiovascular benefit.

The ASCEND trial evaluated the efficacy of aspirin in the primary prevention of CVD in patients with diabetes mellitus. It enrolled a total of 15,480 patients with confirmed diabetes with a mean age of 63 and mean followup of 7.4 years. The primary efficacy outcome was the first serious vascular event, defined as a composite of nonfatal MI, nonfatal stroke, transient ischemic attack, and death from any vascular cause. The primary safety outcome was the first occurrence of any major bleeding, intracranial hemorrhage, sight-threatening bleeding event in the eye, GI bleeding, and any bleeding which required hospitalization and/or transfusion. In contrast to the previous two trials, the ASCEND showed a significant benefit for aspirin. Patients receiving aspirin had a significant reduction in vascular events when compared to placebo $(8.5 \%$ vs. $9.6 \%$; $\mathrm{P}=0.01)$ in the follow-up period. This however, was offset by an increase in major bleeding events (4.2\% in the aspirin group vs. $3.2 \%$ in the placebo; $\mathrm{P}=0.003)$. Similar to ASPREE, the majority of major bleeds were GI which accounted for just shy of half of the events.

ARRIVE, ASCEND, and ASPREE showed that aspirin had at best a minimal CVD benefit with a significant bleeding risk in various patient populations including moderate risk of CVD, elderly patients, and diabetics, respectively. Furthermore, it is difficult to argue that this new data is not valid in the pre-text of older studies that supported and showed the benefit for aspirin. Mahmoud et al. recently published a meta-analysis that combined these recent studies with the prior data available for aspirin in primary prevention. Their analysis included a total of 11 studies with 157,248 patients without pre-existing CVD comparing aspirin $v s$. no aspirin/placebo (10). They found no difference in cardiovascular mortality, other than a slight decrease in MI. However, the reduction in MI was not significant when analysis was limited to studies done after the year 2000. In both the older and contemporary analyses, aspirin use resulted in significant increases in both major bleeding $[1.8 \%$ vs. $1.2 \%$; risk ratio (RR) 1.47 ; $95 \%$ CI, 1.31-1.65; $\mathrm{P}<0.0001]$ and intracranial hemorrhage (0.4\% vs. $0.3 \%$; RR $1.33 ; 95 \%$ CI, $1.13-1.58 ; \mathrm{P}=0.001$ ). This significant increase in major bleeding with aspiring was further validated by another large scale meta-analysis by Huang et al. which was specifically powered to analyze bleeding outcomes (11). The significantly increased risk of intracranial hemorrhage is of particular importance because it carries a 30 -day mortality rate of $35-52 \%$ with only about $20 \%$ of patients with full functional recovery at six months $(12,13)$.

When the data above is placed into context, it is easy to draw the conclusion that aspirin is not as innocuous as 
it was once believed to be, and its bleeding risk should be taken into careful consideration. While the past year does not bode well for the use of aspirin for primary prevention, it remains essential in secondary prevention for patients with established CVD (14). For providers, the difference in primary and secondary prevention indications is clear, but for patients it can be a difficult task. As the above research and guideline changes emerged over the past year, patients were exposed to a variety of misleading article titles such as "don't take an aspirin a day to prevent heart attacks and strokes: doctors reverse recommendation" (15), "study: risks of taking daily, low-dose aspirin outweigh benefits.”(16), and "Taking aspirin daily causes risk of bleeding to increase by 43 percent" (17). This can lead to confusion for patients in whom aspirin therapy may actually be essential: those with established CVD. This is confounded by the fact that aspirin remains OTC often under the marketing of "Heart Healthy" labeled on the bottle itself. With the robust information made available in the past year about the limited benefit in patients without CVD and increased serious bleeding in that subset, it is essential for providers and patients to have effective communication when it comes to the use of aspirin. Moving to a prescription-only model for aspirin would be beneficial in three ways. First, it would allow patients with CVD to discuss with providers why it is included in their therapy and appropriate specifically for them. Second, it would lead providers to reconsider aspirin in the patients under their care without CVD who may have been already taking the medicine or be a new candidate. Finally, it would bring increased awareness to providers for patients who may have been buying the medicine OTC without a full understanding of the significant bleeding risk attributed to aspirin.

In conclusion, more recent studies have now shown that the benefits of aspirin in the primary prevention of cardiovascular events is limited and may be accompanied by potential side effects such as bleeding. Even, among those with established CVD, close monitoring is ideal to avoid bleeding risks. Moving to a prescription only model for the medication would allow close monitoring of patients already on aspirin and allow for providers to take a closer look into patients who may previously be buying the medication OTC and select those who are actually going to benefit from its use.

\section{Acknowledgments}

None.

\section{Footnote}

Conflicts of Interest: Dr. Cave discloses that he serves on the speakers bureau for Portola Pharmaceuticals. The other authors have no conflicts of interest to declare.

Ethical Statement: The authors are accountable for all aspects of the work in ensuring that questions related to the accuracy or integrity of any part of the work are appropriately investigated and resolved.

\section{References}

1. Sneader $\mathrm{W}$. The discovery of aspirin: a reappraisal. BMJ 2000;321:1591-4.

2. Connolly SJ, Milling TJ Jr, Eikelboom JW, et al. Andexanet Alfa for Acute Major Bleeding Associated with Factor Xa Inhibitors. N Engl J Med 2016;375:1131-41.

3. Kaatz S, Bhansali H, Gibbs J, et al. Reversing factor Xa inhibitors - clinical utility of andexanet alfa. J Blood Med 2017;8:141-9.

4. Arnett DK, Blumenthal RS, Albert MA, et al. 2019 ACC/AHA Guideline on the Primary Prevention of Cardiovascular Disease: Executive Summary: A Report of the American College of Cardiology/American Heart Association Task Force on Clinical Practice Guidelines. J Am Coll Cardiol 2019. [Epub ahead of print].

5. January CT, Wann LS, Calkins H, et al. 2019 AHA/ ACC/HRS Focused Update of the 2014 AHA/ACC/ HRS Guideline for the Management of Patients With Atrial Fibrillation: A Report of the American College of Cardiology/American Heart Association Task Force on Clinical Practice Guidelines and the Heart Rhythm Society. J Am Coll Cardiol 2019;74:104-32.

6. Piepoli MF, Hoes AW, Agewall S, et al. 2016 European Guidelines on cardiovascular disease prevention in clinical practice: The Sixth Joint Task Force of the European Society of Cardiology and Other Societies on Cardiovascular Disease Prevention in Clinical Practice (constituted by representatives of 10 societies and by invited experts)Developed with the special contribution of the European Association for Cardiovascular Prevention \& Rehabilitation (EACPR). Eur Heart J 2016;37:2315-81.

7. McNeil JJ, Nelson MR, Woods RL, et al. Effect of Aspirin on All-Cause Mortality in the Healthy Elderly. N Engl J Med 2018;379:1519-28.

8. ASCEND Study Collaborative Group, Bowman L, Mafham M, et al. Effects of Aspirin for Primary 
Prevention in Persons with Diabetes Mellitus. N Engl J Med 2018;379:1529-39.

9. Gaziano JM, Brotons C, Coppolecchia R, et al. Use of aspirin to reduce risk of initial vascular events in patients at moderate risk of cardiovascular disease (ARRIVE): a randomised, double-blind, placebo-controlled trial. Lancet 2018;392:1036-46.

10. Mahmoud AN, Gad MM, Elgendy AY, et al. Efficacy and safety of aspirin for primary prevention of cardiovascular events: a meta-analysis and trial sequential analysis of randomized controlled trials. Eur Heart J 2019;40:607-17.

11. Huang WY, Saver JL, Wu YL, et al. Frequency of Intracranial Hemorrhage With Low-Dose Aspirin in Individuals Without Symptomatic Cardiovascular Disease: A Systematic Review and Meta-analysis. JAMA Neurol 2019. [Epub ahead of print].

12. Caceres JA, Goldstein JN. Intracranial hemorrhage. Emerg Med Clin North Am 2012;30:771-94.

13. Broderick J, Connolly S, Feldmann E, et al. Guidelines for the management of spontaneous intracerebral hemorrhage in adults: 2007 update: a guideline from the American Heart Association/American Stroke Association Stroke Council, High Blood Pressure

Cite this article as: Rawal A, Cave B, Ardeshna D, Hana D, Ibebuogu UN, Khouzam RN. The death of aspirin for primary prevention-should aspirin be changed to a prescription only medication? Ann Transl Med 2019;7(17):402. doi: 10.21037/ atm.2019.07.05
Research Council, and the Quality of Care and Outcomes in Research Interdisciplinary Working Group. Stroke 2007;38:2001-23.

14. Antithrombotic Trialists' (ATT) Collaboration, Baigent C, Blackwell L, et al. Aspirin in the primary and secondary prevention of vascular disease: collaborative meta-analysis of individual participant data from randomised trials. Lancet 2009;373:1849-60.

15. May A. Don't take an aspirin a day to prevent heart attacks and strokes: Doctors reverse recommendation. USA Today. 2019 March 19, 2019. Available online: https:// www.usatoday.com/story/news/health/2019/03/18/aspirinprevent-heart-attacks-strokes-doctors/3199831002/

16. WTNH.com staff. Study: Risks of taking daily, low-dose aspirin outweigh benefits. 2019, January 22. Available online: https://www.wtnh.com/news/health/study-risks-oftaking-daily-low-dose-aspirin-outweigh-benefits/

17. McDermott $\mathrm{N}$. Taking a daily aspirin causes the risk of major bleeding to increase by 43 per cent, study claims. The Sun. 2019, January 23. Available online: https:// www.thesun.co.uk/news/8258114/daily-aspirin-majorbleeding-risk/ 